\title{
Resistencia cardiorrespiratoria y características morfológicas en jugadores de goalball \\ Cardiorespiratory fitness and morphological characteristics in goalball players
}

*Andrés Godoy-Cumillaf, **Nicolás Fica Ramírez, *Paola Fuentes-Merino.

Universidad Autónoma de Chile (Chile), **Instituto Nacional de Deportes (Chile)

\begin{abstract}
Resumen Dentro de las actividades deportivas que practican las personas con discapacidad visual se encuentra el goalball, deporte que ha tenido una gran masificación en el mundo entero, sin embargo la información sobre los beneficios que trae para la salud de quienes lo practican es aún limitada. El objetivo de esta investigación es generar información de la resistencia cardiorrespiratoria (RCR), características morfológicas, de personas discapacitadas visuales que practiquen este deporte. Se trabajó con una muestra de 34 deportistas discapacitados visuales. Las variables estudiadas fueron, RCR, composición corporal, índice de masa corporal (IMC), índice cintura-cadera (ICC). Los resultados indican que la muestra estudiada presenta para RCR valores de 33.6 mililitros por kilogramo de peso por minuto, un 29,6\% para el componente adiposo, un $42.6 \%$ para el muscular, un somatotipo de 4.8 - 5.3 - 1.5, un IMC de 25.6 y un ICC de 0.9. En conclusión, los discapacitados visuales evaluados que practican goalball presentan adecuados valores de RCR. Se sugiere la realización de ensayos clínicos con esta población para determinar los efectos que trae este deporte en la salud de quienes lo practican.
\end{abstract}

Palabras Claves: Composición Corporal, Resistencia Cardiorrespiratoria, Goalball.

Summary Within the sports activities practiced by people with visual disabilities is goalball, a sport that has had a great massification throughout the world, however the information on the benefits it brings to the health of those who practice it is still limited. The objective of this research is to generate information on cardiorespiratory fitness (CRF), morphological characteristics, of visually impaired people who practice this sport. We worked with a sample of 34 visually impaired athletes. The variables studied were, CRF, body composition, body mass index (BMI), waist-hip ratio (WHR). The results indicate that the studied sample presents for CRF values of 33.6 milliliters per kilogram of weight per minute, 29.6\% for the adipose component, $42.6 \%$ for the muscle, a somatotype of $4.8-5.3-1.5$, a BMI of 25.6 and an ICC of 0.9 . In conclusion, the evaluated visually impaired who practice goalball present adequate CRF values. Clinical trials with this population are suggested to determine the effects of this sport on the health of those who practice it.

Key words: Body Composition, Cardiorespiratory Fitness, Goalball.

\section{Introducción}

Más de 1300 millones de personas en el mundo poseen discapacidad visual, la cual puede ir desde una pérdida total de la visión a una reducción moderada, deficiente o grave, tanto para mirar de cerca como de lejos (WHO, 2017), situación que provoca un descenso de la calidad de vida (Ramrattan et al., 2001) y mayor riesgo de mortalidad (McCarty, Nanjan, \& Taylor, 2001). La discapacidad visual conlleva problemas en la orientación espacial, lo que deriva en una reducción de la cantidad de actividad física que se realiza (Bednarczuk et al., 2016). Una investigación desarrollada por Skaggs \& Hopper (1996), que comparó varios estudios en donde se evaluaba la condición física de niños, concluyó que la resistencia cardiorrespiratoria [RCR] y composición

Fecha recepción: 06-04-21. Fecha de aceptación: 24-01-22

Andrés Godoy Cumillaf

andres.godoy@uautonoma.cl corporal de quienes presentan discapacidad visual, sin importar el grado de ésta, evidencia valores más bajos que sujetos de la misma edad, que no poseen la discapacidad. Resultados similares documentaron Lieberman \& McHugh (2001), pero esta vez en niños y adolescentes, lo cual se relaciona con un aumento en los niveles de tejido adiposo, disminución del tejido muscular y baja RCR.

Uno de los deportes que practican quienes sufren discapacidad visual es el goalball, el cual se creó posterior a la segunda guerra mundial con el objetivo de ayudar a la rehabilitación de veteranos de guerra y que en la actualidad es practicado alrededor del mundo entero en más de 112 países (IBSA, 2017). Un estudio realizado por Karakaya \& Nevin (2009) reporta valores más altos de RCR en adolescentes discapacitados visuales que practican este deporte al ser comparados con sujetos sedentarios de su misma edad. Por su parte Caliskan et al. (2011) reportaron reducciones del tejido adiposo al evaluar a niños deficientes visuales que 
fueron sometidos a una intervención de actividad física, siendo el goalball el medio utilizado. Así también Valdés, Godoy \& Herrera (2014), encontraron valores aceptables de composición corporal en adultos que practicaban el deporte. Como se aprecia, la información evidencia beneficios para las personas que lo practican, pero aún es limitada, en especial lo que concierne a RCR, teniendo en cuenta que bajos valores de esta capacidad se encuentran relacionados con la adquisición de enfermedades cardiovasculares (Lin et al., 2015).

Debido a que es necesario generar más información acerca del goalball y los beneficios que trae para la salud, lo que podría estimular una práctica más habitual de este deporte. El objetivo de la presente investigación es determinar resistencia cardiorrespiratoria y características morfológicas de jugadores de goalball.

\section{Material y método}

Investigación no experimental, descriptiva, transversal, con enfoque cuantitativo, con un muestreo no probabilístico por conveniencia.

\section{Sujetos}

Se evaluó a 34 jugadores de goalball masculinos discapacitados visuales, con edades entre los 25 a 45 años. Los sujetos fueron reclutados de 6 equipos de goalball de Chile. Los criterios para la inclusión en este estudio fueron: a) presentar ceguera total (B1) o ceguera con restos visuales (B2 y B3) (IBSA, 2017); b) durante el último año, encontrarse entrenando 3 veces por semana; y c) cumplir con la firma de un consentimiento informado que autoriza el uso de la información con fines científicos. Las evaluaciones se realizaron el día previo a un encuentro nacional, en el mes de noviembre del año 2019, en la ciudad de Santiago de Chile. El estudio fue aprobado por el comité ético de la Universidad $\mathrm{Au}$ tónoma de Chile ( $\left.\mathrm{N}^{\circ} 041-15\right)$ y desarrollado siguiendo lo expuesto en la declaración de Helsinki.

\section{Instrumentos}

La RCR se evaluó por medio de la prueba de carrera de 20 metros (Léger, Mercier, Gadoury \& Lambert, 1988), donde los participantes debían correr entre dos marcas separadas entre sí por dicha distancia, llegando a estas marcas junto con una señal de audio emitida desde un disco compacto pregrabado. La velocidad inicial fue de $8,5 \mathrm{~km} / \mathrm{hr}$, la que se incrementaba $1 \mathrm{~km} / \mathrm{hr}$ cada un minuto. La prueba finaliza cuando el evaluado no puede alcanzar la marca al mismo tiempo que la señal de audio.
Se registró la última etapa completada, dato que ingresado a la fórmula propuesta por Léger et al., entrega como resultado el consumo de oxígeno (Vo2máx), el cual se expresa en mililitros por kilogramo de peso por minuto $(\mathrm{ml} / \mathrm{kg} / \mathrm{min})$. Para la realización de esta prueba, cada uno de los evaluados fue acompañado durante todo el transcurso, por una persona que le indicaba la marca donde se debía detener, girar y correr en dirección a la otra marca. La clasificación de los valores se basó en los puntos de referencia usados en adultos (American College of Sports Medicine, 2013).

La composición corporal se estimó mediante el método de 5 componentes que divide al cuerpo en tejido adiposo, muscular, óseo, residual y piel (Kerr, 1988), el somatotipo a través del método de Heath \& Carter (1990). Se calcularon además el índice de masa corporal (IMC) dividiendo el peso por la talla al cuadrado, para una posterior clasificación del estado nutricional de los evaluados según lo recomendado por la Organización Mundial de la Salud (WHO, 2011), el índice cintura-cadera (ICC) se determinó dividiendo el perímetro de cintura por el perímetro de cadera, para clasificar según lo recomendado por WHO (2011), si tiene o no riesgo de adquirir enfermedades metabólicas. La suma de seis pliegues cutáneos en mm (tricipital, subescapular, cresta iliaca, abdominal, muslo y pantorrilla) y la relación músculo-hueso dividiendo $\mathrm{kg}$ de músculo por $\mathrm{kg}$ de hueso. Todas las evaluaciones fueron realizadas por un antropometrista nivel 3, error de medición para todas las variables de 1,2\%. Las medidas fueron tomadas siguiendo el protocolo expuesto por la Sociedad Internacional para el Avance de la Kineantropometría (Marfell-Jones, 2012). La masa corporal se evaluó con balanza digital (Scale-tronik $k_{\mathbb{B}}$, USA) con precisión de $0,1 \mathrm{~kg}$. La altura con estadiómetros (Seca $220_{\circledast}$, Alemania), con precisión de $1 \mathrm{~mm}$. Para la altura sentado se utilizó estadiómetro (Seca 220 $0_{\circledast}$, Alemania), además de cajas de madera de $50 \mathrm{~cm}$ de altura. Los diámetros de mayor tamaño fueron medidos con antropómetros Campbell 20 $0_{\circledast}$ (Rosscraft, Buenos Aires), y los de menor tamaño con antropómetros Campbell 10 $0_{\circledast}$ (Rosscraft, Buenos Aires), ambos con precisión de $1 \mathrm{~mm}$. Los perímetros corporales con cinta metálica no extensible (Rosscraft ${ }_{\mathbb{B}}$, Canadá) precisión $1 \mathrm{~mm}$ y los pliegues cutáneos con calibres (Harpender ${ }_{\mathbb{B}}$, Reino Unido) precisión $2 \mathrm{~mm}$. Todas las medidas fueron tomadas por duplicado.

En lo que respecta a análisis estadístico, en todas las variables estudiadas se calculó media aritmética, desviación estándar, valores mínimos, máximos e interva- 
los de confianza al 95\%. Para determinar la normalidad de las variables se utilizó la prueba de Shapiro-wilk. Los resultados fueron obtenidos con el paquete estadístico SPSS 25.0.

\section{Resultados}

La tabla 1 entrega los valores obtenidos en las variables estudiadas. Para RCR la media fue de $33.6 \mathrm{ml} / \mathrm{kg} /$ min. Para los componentes de tejido adiposo, muscular y óseo presentan una media de 29.6\%, 42.6\% y 10.6\%, respectivamente. Un somatotipo que los clasifica como

\begin{tabular}{|c|c|c|c|c|}
\hline & Mínimo & Media \pm DS & Máximo & $95 \%$ IC \\
\hline \multicolumn{5}{|l|}{ Adiposa } \\
\hline$\%$ & 18.6 & $29.6 \pm 5.4$ & 37.5 & $27.7-31.5$ \\
\hline $\mathrm{Kg}$ & 10.5 & $21.2 \pm 5.3$ & 30.3 & $19.3-23.1$ \\
\hline \multicolumn{5}{|l|}{ Muscular } \\
\hline$\%$ & 34.8 & $42.6 \pm 4.4$ & 49.6 & $41.1-44.2$ \\
\hline $\mathrm{Kg}$ & 18.6 & $31.2 \pm 7.6$ & 51.4 & $28.5-33.9$ \\
\hline \multicolumn{5}{|l|}{ Residual } \\
\hline$\%$ & 9.4 & $12 \pm 1.2$ & 14.2 & $11.5-12.4$ \\
\hline $\mathrm{Kg}$ & 6.5 & $8.7 \pm 2.2$ & 15.7 & $7.9-9.5$ \\
\hline \multicolumn{5}{|l|}{ Óseo } \\
\hline$\%$ & 8.9 & $10.6 \pm 1.8$ & 17 & $10-11.3$ \\
\hline $\mathrm{Kg}$ & 7.1 & $8.3 \pm 0.9$ & 10.2 & $8-8.6$ \\
\hline \multicolumn{5}{|l|}{ Piel } \\
\hline$\%$ & 3.8 & $4.9 \pm 0.6$ & 5.8 & $4.7-5.1$ \\
\hline $\mathrm{Kg}$ & 2.9 & $3.5 \pm 0.4$ & 4.6 & $3.3-3.7$ \\
\hline \multicolumn{5}{|l|}{ Somatotipo } \\
\hline Endomorfía & 1.8 & $4.8 \pm 1.5$ & 7.6 & $4.2-5.3$ \\
\hline Mesomorfía & 2.2 & $5.3 \pm 1.6$ & 8.5 & $4.7-5.9$ \\
\hline Ectomorfía & 0.5 & $1.5 \pm 0.7$ & 4.7 & $1.1-1.9$ \\
\hline \multicolumn{5}{|l|}{ Índices } \\
\hline $\operatorname{IMC}\left(\mathrm{kg} / \mathrm{m}^{2}\right)$ & 18.6 & $25.6 \pm 3.9$ & 33.2 & $24.3-27$ \\
\hline $\mathrm{M} / \mathrm{H}$ & 2.4 & $4 \pm 0.6$ & 5.3 & $3.8-4.3$ \\
\hline ICC & 0.7 & $0.9 \pm 0.1$ & 1 & $0.8-0.9$ \\
\hline Suma6 & 64 & $81.5 \pm 24.7$ & 99 & $79.2-83.1$ \\
\hline \multicolumn{5}{|l|}{ Resistencia cardiorrespiratoria } \\
\hline $\mathrm{ml} / \mathrm{kg} / \mathrm{min}$ & 26.6 & $33.6 \pm 4.1$ & 41.6 & $32.2-35.1$ \\
\hline \multicolumn{5}{|c|}{ IMC: Índice de masa corporal. M/H: Relación músculo-hueso. } \\
\hline \multicolumn{5}{|c|}{ ICC: Índice Cintura Cadera. Suma 6: Suma de 6 pliegues cutáneos. } \\
\hline \multirow{2}{*}{\multicolumn{5}{|c|}{$\begin{array}{l}\text { Vo2máx: Consumo de oxígeno. } \mathrm{ml} / \mathrm{kg} / \mathrm{min} \text { : Mililitros por kilogramo de peso por minuto. } \\
\mathrm{Kg} / \mathrm{m}^{2} \text { : Kilogramos por metro cuadrado. }\end{array}$}} \\
\hline & & & & \\
\hline \multicolumn{5}{|c|}{ Tabla 2.} \\
\hline \multicolumn{5}{|c|}{ Variables antropométricas estimadas en los deportistas evaluados. } \\
\hline & Mínimo & Media \pm DS & Máximo & $95 \%$ IC \\
\hline Peso $(\mathrm{kg})$ & 57 & $72.8 \pm 12.4$ & 109 & $68.5-77.2$ \\
\hline Talla $(\mathrm{cm})$ & 157 & $169.4 \pm 7$ & 181 & $166.9-171.8$ \\
\hline Talla sentado $(\mathrm{cm})$ & 77 & $86.1 \pm 5$ & 96 & $84.3-87.8$ \\
\hline \multicolumn{5}{|l|}{ Diámetros } \\
\hline Biacromial $(\mathrm{cm})$ & 36 & $39 \pm 1.8$ & 44 & $28.1-29.8$ \\
\hline Tórax Transverso (cm) & 17 & $28.9 \pm 2.3$ & 26 & $28.1-29.8$ \\
\hline Tórax anteroposterior $(\mathrm{cm})$ & 17 & $21.2 \pm 2.5$ & 26 & $20.2-22$ \\
\hline Bi-iliocrestideo $(\mathrm{cm})$ & 26 & $27.8 \pm 2$ & 33 & $27.1-28.6$ \\
\hline Humeral $(\mathrm{cm})$ & 6 & $6.7 \pm 0.4$ & 8 & $6.6-6.9$ \\
\hline Femoral $(\mathrm{cm})$ & 9 & $9.4 \pm 0.6$ & 11 & $9.2-9.6$ \\
\hline Perímetros & & & & \\
\hline Cabeza $(\mathrm{cm})$ & 51 & $57.8 \pm 10$ & 97 & $54.3 \pm 61.3$ \\
\hline Brazo relajado $(\mathrm{cm})$ & 26 & $30.5 \pm 3.7$ & 39 & $29.2-31.8$ \\
\hline Brazo contraído $(\mathrm{cm})$ & 27 & $32.5 \pm 3.6$ & 41 & $31.3-33.8$ \\
\hline Antebrazo $(\mathrm{cm})$ & 22 & $26.3 \pm 2.4$ & 32 & $25.5-27.2$ \\
\hline Tórax mesoesternal $(\mathrm{cm})$ & 88 & $100.1 \pm 9.8$ & 128 & $96.6-103.5$ \\
\hline Cintura $(\mathrm{cm})$ & 77 & $88.6 \pm 10.3$ & 115 & $85-92.2$ \\
\hline Cadera $(\mathrm{cm})$ & 89 & $99.2 \pm 14.1$ & 148 & $94.3-104.2$ \\
\hline Muslo máximo (cm) & 49 & $55.8 \pm 4.3$ & 65 & $54.3-57.4$ \\
\hline Muslo medio (cm) & 47 & $51.4 \pm 3.6$ & 61 & $50.2-52.7$ \\
\hline Pantorrilla $(\mathrm{cm})$ & 30 & $36 \pm 3.2$ & 44 & $34.9-37.1$ \\
\hline $\begin{array}{l}\text { Pliegues } \\
\end{array}$ & & & & \\
\hline Tricipital (mm) & 6 & $11.7 \pm 3.2$ & 17 & $10.6-12.8$ \\
\hline Subescapular $(\mathrm{mm})$ & 5 & $18.8 \pm 9.5$ & 41 & $15.5-22.1$ \\
\hline Supraespinal $(\mathrm{mm})$ & 8 & $18.2 \pm 10.7$ & 53 & $14.5-22$ \\
\hline Abdominal (mm) & 8 & $26.4 \pm 10.9$ & 56 & $22.6-30.2$ \\
\hline Muslo (mm) & 10 & $16.5 \pm 5.3$ & 27 & $14.7-18.4$ \\
\hline Pantorrilla (mm) & 3 & $11.1 \pm 5.7$ & 22 & $9.1-13.1$ \\
\hline $\begin{array}{l}\text { Tabla } 3 . \\
\text { Clasificaciones para consumo de oxí } \\
\text { evaluados. }\end{array}$ & xigeno, índice de $n$ & a corporal e ind & cintura ca & de los deportistas \\
\hline Vo2máx. & & IMC & & ICC \\
\hline Inadecuado $\quad$ Adecuado & Normal & obrepeso/obesidad & Con riesg & o Sin riesgo \\
\hline $49 \% \quad 51 \%$ & $53 \%$ & $47 \%$ & $56 \%$ & $44 \%$ \\
\hline
\end{tabular}

mesoendomorfos balanceados (4.8 - 5.3-1.5), un IMC con una media de 25.6 y un ICC con un valor de 0.9. También hay información sobre la suma de seis pliegues cutáneos, somatotipo y relación músculo-hueso, la cual puede ser útil, para investigadores o entrenadores, con fines de comparación. Los valores medios de todas las variables antropométricas estimadas se pueden observar en la tabla 2 .

La tabla 3 muestra clasificaciones, encontrándose un $51 \%$ de evaluados clasificado como adecuado para RCR, un 53\% de los evaluados en un estado normal para el IMC, un 56\% con riesgo para el ICC.

\section{Discusión}

El presente estudio aporta evidencia la cual indica que el $51 \%$ de los discapacitados visuales evaluados que practican goalball obtienen valores adecuados de RCR, y que esta se encuentra relacionada de manera positiva con tejido muscular y negativa con tejido adiposo.

El IMC promedio encontrado en los evaluados (25.6 $\mathrm{kg} / \mathrm{m}^{2}$ ) es ligeramente más bajo a lo reportado en sujetos de similares características y misma nacionalidad $\left(26 \mathrm{~kg} / \mathrm{m}^{2}\right)$ (Valdés et al.), pero superior a lo indicado en discapacitados visuales de España que practican deportes adaptados de características similares $(23.1 \mathrm{~kg} /$ $\mathrm{m}^{2}$ ) (Torralba et al., 2015) y a deportistas brasileros de nivel paraolímpico $\left(23 \mathrm{~kg} / \mathrm{m}^{2}\right)$ (Scherer et al., 2012). En relación al estado nutricional, debido a los escasos estudios encontrados se hace difícil realizar comparaciones con población similar, pero existe evidencia que un $63 \%$ de la población chilena y un $58 \%$ de latinoamericana se encuentran con sobrepeso u obesidad (FAO, 2017), mientras que un $47 \%$ de los evaluados se clasifica de igual manera, lo que refleja que quienes practican goalball presentan mejores valores, lo que podría ayudar en la prevención y/o tratamiento del sobrepeso y obesidad.

En lo que respecta al ICC, los evaluados obtienen un valor promedio de 0.9 , el cual es más bajo a lo reportado en deportistas de características similares (0.96) (Valdés et al.), pero mayor al encontrado en sujetos de menor edad (21.7 años), sin discapacidad y con un alto nivel de actividad física, cuyos valores fueron de 0,76 (Salvador, Godoy, Mella, López y Fernández, 2016). En cuanto a clasificaciones, un $56 \%$ se encuentra con riesgo de adquirir enfermedades metabólicas (índice ICC), situación preocupante ya que existe evidencia consistente que respalda al ICC como una medida de adiposidad abdominal y de riesgo de adquirir enfermedades 
cardiovasculares y diabetes tipo 2, debido a que el aumento del tejido adiposo visceral se asocia con un rango de anomalías metabólicas, incluida la disminución de la tolerancia a la glucosa, la reducción de la sensibilidad a la insulina y los perfiles lipídicos adversos (Huxley, Mendis, Zheleznyakov, Reddy \& Chan, 2010; Qiao \& Nyamdorja, 2010a; Qiao \& Nyamdorj, 2010b; Seidell, 2010).

Para RCR, los deportistas obtienen un valor promedio de $33,6 \mathrm{ml} / \mathrm{kg} / \mathrm{min}$, lo que se encuentra por debajo de lo reportado en sujetos físicamente activos, como lo son estudiantes universitarios de educación física y jugadores adultos de voleibol, quienes obtienen valores promedios de $42.84 \mathrm{ml} / \mathrm{kg} / \mathrm{min}$ (Almagia, Lizana, Rodriguez, Ivanovic y Binvignat, 2009) y 52.99 $\mathrm{ml} / \mathrm{kg} / \mathrm{min}$ (Kausar, Mudassir, Badaam, Shete \& Khan, 2015) respectivamente. Pero similares a lo encontrado en discapacitados visuales practicantes de goalball, cuyo valor promedio fue de $34.7 \mathrm{ml} / \mathrm{kg} / \mathrm{min}$ (Gulick \& Malone, 2011). Al comparar con sujetos sedentarios, se ven semejanzas con jóvenes de 22,2 años de edad promedio, quienes presentan $37.01 \mathrm{ml} / \mathrm{kg} / \mathrm{min}$ (Kausar et al.), y se evidencian mejores valores al comparar con adultos sedentarios quienes reportan $24 \mathrm{ml} / \mathrm{kg} / \mathrm{min}$ (Taub et al., 2016) lo que concuerda con lo planteado por López y Fernández (2006), quienes plantean que la RCR puede ser modificada por factores como el entrenamiento y la edad. Estos resultados confirman al goalball como un medio que podría ayudar a mejorar los valores. No obstante, estos datos difieren absolutamente con Skaggs \& Hopper, quienes analizaron 11 estudios que estudiaron a discapacitados visuales, concluyendo que sin importar el grado de la discapacidad, su RCR es más baja que sujetos de la misma edad sin discapacidad visual. Cobrando fuerza lo planteado por Karakaya \& Nevin, al decir que es importante dirigir a las personas con discapacidad visual a la participación en deportes o actividades recreativas para mejorar su condición física.

En cuanto a composición corporal, el tejido muscular fue de $42.6 \%$ y el adiposo de $29.6 \%$, valores similares a deportistas de goalball de igual nivel de competición (42.7\% muscular, 28.7\% adiposo) (Valdés et al.), pero que al ser contrastados con atletas brasileros de nivel paraolímpico (Scherer et al.), son más bajos para el tejido muscular (45.5\%) y similares para el adiposo (28.7\%). Mientras que al comparar con tablas de referencia para sujetos chilenos físicamente activos (Rodríguez et al., 2010), se clasifican como superior a la norma, lo cual sugiere nuevamente al goalball como medio que ayuda a mejorar la salud de quienes lo practican, en especial a lo que se refiere a tejido muscular, debido a que valores aceptables o superiores de este componente beneficia ante enfermedades cardiovasculares, producto del factor protector que ejerce la masa muscular ante este tipo de padecimientos (Srikanthan et al., 2016).

Todos los antecedentes encontrados, evidencian a una gran cantidad de evaluados con sobrepeso, obesidad y riesgo metabólico, situándolos en una situación preocupante debido a la relación que tienen el IMC y el ICC con un aumento del riesgo de mortalidad (Telles, Kala, Sharma \& Balkrishna, 2018;WHO, 2011), pero por otro lado son alentadores debido a que demuestran que la práctica del deporte podría ser un medio que ayude a mejorar los valores de RCR y tejido muscular, lo cual contribuiría a reducir el riesgo que generan altos valores de IMC e ICC (Lin et al., 2015). Además de lo anterior, el que sean sujetos físicamente activos, los ayuda a tener menor riesgo de mortalidad por eventos cardiovasculares, a pesar de obtener altos valores de IMC o tejido adiposo, debido al factor protector que provoca la actividad física (McAuley et al., 2012)

Las principales fortalezas de esta investigación están en: 1) generar información sobre características morfológicas e índices evaluados, la cuales pueden ser de utilidad para entrenadores o investigadores que deseen realizar comparaciones, 2) entregar información sobre una capacidad poco evaluada en estos deportistas RCR. Las limitaciones vienen dadas por ser una investigación de carácter transversal, lo cual no permite determinar los beneficios que trae el deporte en los evaluados. En este sentido, por medio de ensayos clínicos, futuras investigaciones deben indagar sobre los efectos que trae este deporte.

\section{Conclusiones}

En conclusión, los discapacitados visuales practicante de goalball evaluados, obtienen adecuados valores de RCR. Situación cual podría ayudar a minimizar el riesgo de mortalidad y adquisición de enfermedades metabólicas, sin embargo, debe ser corroborado a través de ensayos clínicos. Se recomienda por tanto, ampliar la investigación en esta población debido a que ha sido poco estudiada, incluyendo variables que también influyen sobre lo estudiado como la actividad física total diaria. 


\section{Referencias}

Almagia, A., Lizana, P., Rodriguez, F., Ivanovic, D. \& Binvignat, G. (2009). Variables Antropometricas y Rendimiento Fisico en Estudiantes Universitarios de Educacion Fisica. Int.J. Morphol., 27(4), 971-975.

American College of Sports Medicine (ACSM). (2013). ACSM's Guidelines for Exercise Testing and Prescription. Philadelphia, United States, Wolters Kluwer Lippincott Williams \& Wilkins Health.

Bednarczuk, G., Molik, B., Morgulec-Adamowicz, N., Kosmol, A., Wiszomirska, I., Rutkowska, I. \& Perkowski, K. (2017). Static balance of visually impaired paralympic goalball players. International Journal of Sports Science \& Coaching, 12(5), 611-617.

Caliskan, E., Pehlivan, A., Erzeybek, M., Kayap1Õnar, F., Agopyan, A., Yuksel, S. \& Dane, S. (2011). Body mass index and percent body fat in goalball and movement education in male and female children with severe visual impairment. Neurology, Psychiatry and Brain Research, 17, 39-41.

Carter, J. E. L. \& Heath, B. H. (1990). Somatotypingdevelopment and applications. Cambridge University Press, Cambridge, Reino Unido.

FAO. Organización de las Naciones Unidas para la Alimentación y la Agricultura America Latina y el Caribe. (2017). Panorama de la seguridad alimentaria y nutricional. Organizacion de las Naciones Unidas para la Alimentacion y la Agricultura y la Organizacion Panamericana de la Salud. Recuperado de http:// www.fao.org/3/a-i6747s.pdf

Gakidou, E., Afshin, A., Abajobir, A., Abate, K., Abbafati, C., Abbas, K., ... Aboyans, V. (2017). Global, regional, and national comparative risk assessment of 84 behavioural, environmental and occupational, and metabolic risks or clusters of risks, 1990-2016. Lancet, 390(10100), 1345-422.

Huxley, R., Mendis, S., Zheleznyakov, E., Reddy, S. \& Chan, J. (2010). Body mass index, waist circumference and waist:hip ratio as predictors of cardiovascular risk - a review of the literature. European Journal of Clinical Nutrition, 64(1), 1622.

International Blind Sports Federation. (2017). IBSA IClassification. Available at: www.ibsasport.org/ classification (accessed 11 January 2017).

Karakaya, I. \& Nevin, E. (2009). Physical fitness of visually impaired adolescent goalball players. Perceptual and Motors Skills, 108, 129-136.

Kausar, A., Mudassir, S., Badaam, K., Shete, A. \& Khan, S. (2015). Cardiorespiratory Fitness of University
Volleyball Players and Sedentary Young People in Marathwada Region of Maharashtra Province in India. J Clin Diagn Res, 9(7), 20-21.

Kerr, D. (1998). An anthropometric method for fractionation of skin, adipose, bone, muscle and residual tissue masses, in males and females age 6 to 77 years. (Master's Thesis). Simon Fraser University, Vancouver, Canada.

Léger, L., Mercier, D., Gadoury, C. \& Lambert, J. (1988). The multistage 20 metre shuttle run test for aerobic ûtness. J. Sports Sci, 6(2), 93-101.

Lieberman, L. \& McHugh, E. (2001). Health-Related Fitness of Children Who Are Visually Impaired. Journal oflisual Impairment \& Blindness, 95(5), 272-287.

Lin, X., Zhang, X., Guo, J., Roberts, C., McKenzie, S., Wu, W., Liu, S. \& Song, Y. (2015). Effects of Exercise Training on Cardiorespiratory Fitness and Biomarkers of Cardiometabolic Health:A Systematic Review and Meta-Analysis of Randomized Controlled Trials. J. Am. Heart. Assoc, 4 e002014.

Lopez, C. \& Fernandez, V. (2006). Fisiologia del ejercicio. Madrid: Panamericana.

Marfell-Jones, M., Olds, T., Stewart, A. D. \& Carter, L. (2012). International Standards for Anthropometric Assessment. Potchefstroom, Sudáfrica: Society for the Advancement of Kinanthropometry (ISAK).

McAuley, P., Artero, E., Sui, X., Lee, D., Church, T., Lavie, C.,... Blair, S. (2012). The obesity paradox, cardiorespiratory fitness, and coronary heart disease. Mayo Clin Proc, 87(5), 443-51.

McCarty, C., Nanjan, M. \&Taylor, H. (2001). Vision impairment predicts 5 year mortality. $\mathrm{Br} J$ Ophthalmol, 85, 322-326.

Ortega,O., Sui, X., Lavie, C. \& Blair, S. (2016). Body Mass Index, the most widely used but also widely criticized index: would a criterion standard measure of total body fat be a better predictor of cardiovascular disease mortality? Mayo Clin Proc. 91(4), 443-55.

Qiao, Q. \& Nyamdorj, R. (2010a). Is the association of type II diabetes with waist circumference or waist to hip ratio stronger than that with body mass index? European Journal of Clinical Nutrition, 64(1), 3034.

Qiao, Q. \& Nyamdorj, R. (2010b). The optimal cutoff values and their performance of waist circumference and waist to hip ratio for diagnosing type II diabetes. European Journal of Clinical Nutrition, 64(1), 2329.

Ramrattan, R., Wolfs, R., Panda-Jonas, S., Jonas, J., Bakker, D., Pols, H. ,... de Jong, P. (2011). Prevalence and causes of visual field loss in the elderly and 
associations with impairment in daily functioning: the Rotterdam Study. Arch Ophthalmol, (12):17881794.

Rodriguez, R., Almagia, F., Yuing, T., Binvignat, G. \& Lizana, A. (2010). Composicion corporal y somatotipo referencial de sujetos fisicamente activos. Int.J. Morphol, 28(4), 1159-1165.

Salvador, N., Godoy, A., Mella, J., Carmona, M. y Fernández, J. (2016). Estado nutricional, riesgo cardiovascular y nivel de glucemia de estudiantes universitarios de Pedagogia en Educacion Fisica. Nutr. Hosp. 33(2), 289-291.

Scherer, R., Karasiak, F., Silva, S. \& Petroski, E. (2012). Morphological profile of goalball athletes. Mot. Eur.J. Hum. Mov, 28, 1-13.

Seidell, J. (2010). Waist circumference and waist/hip ratio in relation to all cause mortality, cancer and sleep apnea. European Journal of Clinical Nutrition, 64(1), 3541.

Skaggs, S. \& Hopper, C. (1996). Individual with visual impairment: a review of psychomotor behavior. Adapted Physical Activity Quarterly, 13, 16-26.

Srikanthan, P., Horwich, T. \& Tseng, C. (2016). Relation of Muscle Mass and Fat Mass to Cardiovascular Disease Mortality. The American Journal of Cardiology, 117(8), 1355-60.

Taub, P., Ramírez-Sánchez, I., Patel, M., Higginbotham, E., Moreno-Ulloa, A., Román-Pintos, L.,... Villareal, F. (2016). Beneficial effects of dark chocolate on exercise capacity in sedentary subjects:
Underlying mechanisms: A double blind, randomized, placebo controlled trial. Food Funct, 7(9), 3686-3696.

Telles, S., Kala, N., Sharma, S. \& Balkrishna, A. (2018). Anthropometric variables as predictors of aspects of quality of life in persons with central obesity. BMC Res Notes, 11(1), 680-684.

Torralba, M., Vives, J., Braz, M. \& Nikic, M. (2015). Control fisiológico para valorar las capacidades y características de deportistas con discapacidad visual. Apunts Med Esport, 50(187), 85-93.

Valdés, P., Godoy, A. \& Herrera, T. (2014). Somatotipo, Composicion Corporal, Estado Nutricional y Condicion Fisica en Personas con Discapacidad Visual que Practican Goalball. Int.J. Morphol, 32(1), 183189.

Wormser, D. , Kaptoge, S., Di Angelantonio, E., Wood, A., Pennels, L., Thompson, A.,... Danesh, J. (2011). Separate and combined associations of body-mass index and abdominal adiposity with cardiovascular disease: collaborative analysis of 58 prospective studies. Lancet, 377(9771), 1085-95.

World Health Organization (WHO). (2011). Waist Circumference andWaist-Hip Ratio:Report of aWHO Expert Consultation. Geneva, Switzerland, World Health Organization.

World Health Organization (WHO). 2017. Blindness and vision impairment. Recuperado de http:// www.who.int/news-room/fact-sheets / detail/ blindness-and-visual-impairment.
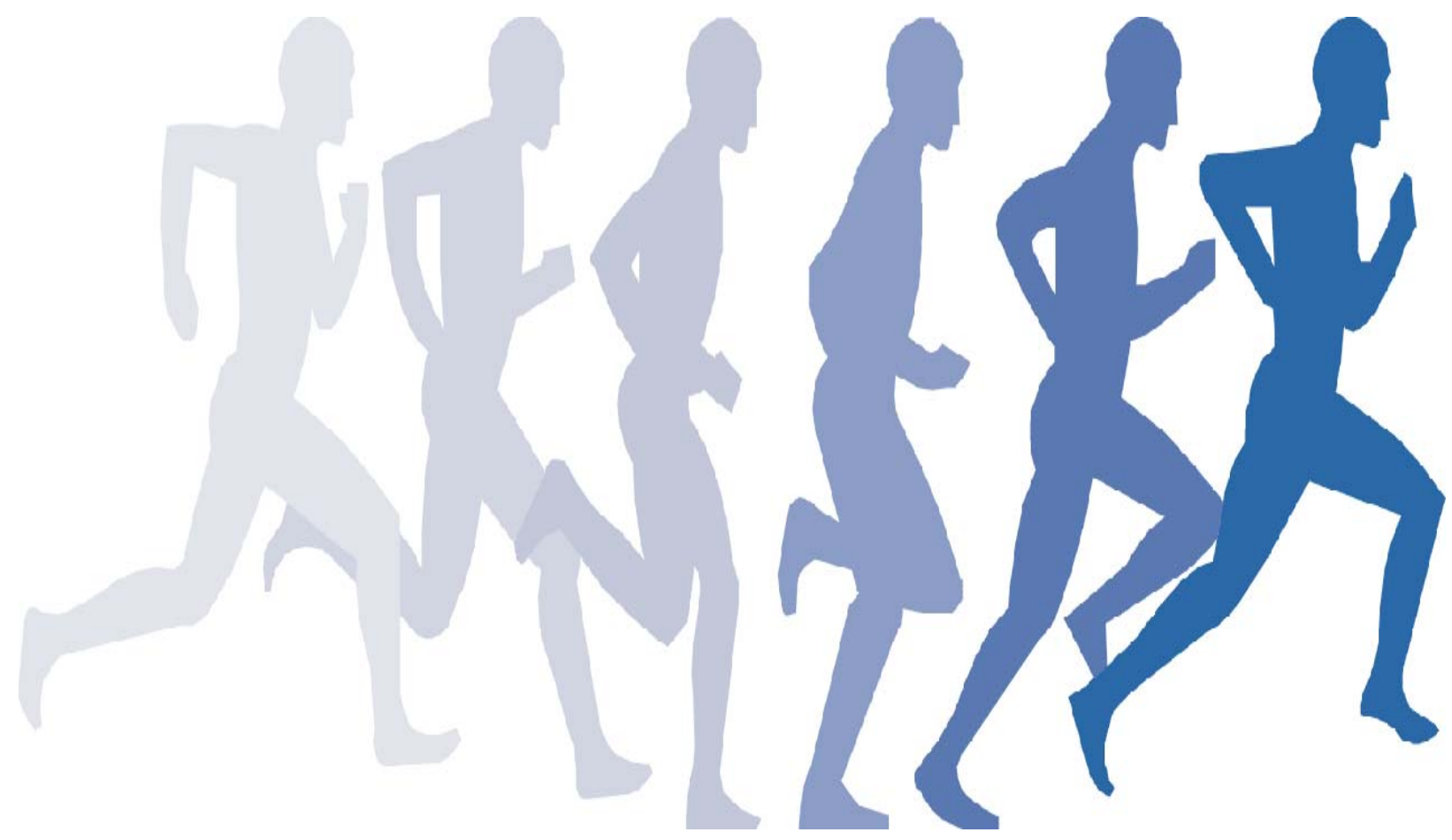